\title{
Macrostructural Changes of the Acoustic Radiation in Humans with Hearing Loss and Tinnitus Revealed with Fixel-Based Analysis
}

\author{
${ }^{\circledR}$ Elouise A. Koops, ${ }^{1,2,3}$ Shereif Haykal, ${ }^{2,3,4}$ and ${ }^{\circledR}$ Pim van Dijk ${ }^{1,2,3}$ \\ ${ }^{1}$ Department of Otorhinolaryngology/Head and Neck Surgery, University Medical Center Groningen, University of Groningen, 9700 RB, Groningen, \\ The Netherlands, ${ }^{2}$ Graduate School of Medical Sciences, Research School of Behavioural and Cognitive Neurosciences, University of Groningen, \\ Groningen, 9713 AV, The Netherlands, ${ }^{3}$ Cognitive Neuroscience Center, Biomedical Sciences of Cells and Systems, University of Groningen, 9713 \\ AW, Groningen, The Netherlands, and ${ }^{4}$ Department of Ophthalmology, University Medical Center Groningen, University of Groningen, 9700 RB, \\ Groningen, The Netherlands
}

Age-related hearing loss is the most prevalent sensory impairment in the older adult population and is related to noiseinduced damage or age-related deterioration of the peripheral auditory system. Hearing loss may affect the central auditory pathway in the brain, which is a continuation of the peripheral auditory system located in the ear. A debilitating symptom that frequently co-occurs with hearing loss is tinnitus. Strikingly, investigations into the impact of acquired hearing loss, with and without tinnitus, on the human central auditory pathway are sparse. This study used diffusion-weighted imaging (DWI) to investigate changes in the largest central auditory tract, the acoustic radiation, related to hearing loss and tinnitus. Participants with hearing loss, with and without tinnitus, and a control group were included. Both conventional diffusion tensor analysis and higher-order fixel-based analysis were applied. The fixel-based analysis was used as a novel framework providing insight into the axonal density and macrostructural morphologic changes of the acoustic radiation in hearing loss and tinnitus. The results show tinnitus-related atrophy of the left acoustic radiation near the medial geniculate body. This finding may reflect a decrease in myelination of the auditory pathway, instigated by more profound peripheral deafferentation or reflecting a preexisting marker of tinnitus vulnerability. Furthermore, age was negatively correlated with the axonal density in the bilateral acoustic radiation. This loss of fiber density with age may contribute to poorer speech understanding observed in older adults.

Key words: acoustic radiation; acquired hearing loss; age; diffusion-weighted imaging; fixel-based analysis; tinnitus

Significance Statement

Age-related hearing loss is the most prevalent sensory impairment in the older adult population. Older individuals are subject to the cumulative effects of aging and noise exposure on the auditory system. A debilitating symptom that frequently cooccurs with hearing loss is tinnitus: the perception of a phantom sound. In this large DWI-study, we provide evidence that in hearing loss, the additional presence of tinnitus is related to degradation of the acoustic radiation. Additionally, older age was related to axonal loss in the acoustic radiation. It appears that older adults have the aggravating circumstances of age, hearing loss, and tinnitus on central auditory processing, which may partly be because of the observed deterioration of the acoustic radiation with age.

Received Nov. 27, 2020; revised Feb. 8, 2021; accepted Mar. 2, 2021.

Author contributions: E.A.K. and P.v.D. designed research; E.A.K. performed research; S.H. contributed unpublished reagents/analytic tools; E.A.K. analyzed data; S.H. and P.v.D. edited the paper; E.A.K. wrote the paper.

This work was supported by the Dorhout Mees Foundation, Daniel Ballinger Memorial Award, British Tinnitus Association, Netherlands Organisation for Scientific Research, American Tinnitus Association, William Demant Foundation, Heinsius Houbolt Foundation, and Steunfonds Audiologie and Stichting Gehoorgestoorde Kind. We thank Prof. Paola Perin for input on the molecular aspects of white matter fibers.

The authors declare no competing financial interests.

Correspondence should be addressed to Elouise A. Koops at e.a.koops@umcg.nl.

https://doi.org/10.1523/JNEUROSCI.2996-20.2021

Copyright $\odot 2021$ the authors

\section{Introduction}

Age-related hearing loss is the most prevalent sensory impairment in older individuals (https:/www.who.int/healthinfo/ statistics/bod_hearingloss.pdf). In hearing loss, the fast and efficient information transfer in the auditory system is hampered by a reduction in hearing sensitivity related to damage of the peripheral auditory pathway. A challenge in defining the characteristic changes of the auditory system related to hearing loss is that hearing loss often co-occurs with other auditory domain conditions. The most extensively studied co-occurring symptom is 
tinnitus, the perception of a phantom sound. Tinnitus is a common and debilitating symptom that affects $\sim 12-30 \%$ of the general population (McCormack et al., 2016). Unlike hearing loss, research investigating damage to the peripheral auditory system in tinnitus is sparse. Available research suggests that tinnitus relates to distinctive damage to peripheral auditory structures (Rüttiger et al., 2013; Singer et al., 2013). In addition to the damage to the structures of the peripheral auditory system, changes to the structure of the central auditory system have been implicated in both hearing loss and tinnitus.

Most of the information transfer in the auditory system takes place via myelinated axons, that is, white matter tracts, which facilitate rapid and precise information transfer (Sinclair et al., 2017). In neuroscience, diffusion-weighted imaging (DWI) is the most commonly used method to investigate white matter tracts of humans with hearing loss and tinnitus. DWI scans, obtained with a magnetic resonance imaging (MRI) scanner, reflect the diffusion of water molecules. In the white matter tracts of the brain, water molecules are most likely to diffuse in parallel with the fibers because of restrictions by axonal membranes and myelination. Traditionally, diffusion-weighted images are analyzed with a diffusion tensor imaging (DTI) model. Within this model, white matter is quantified by the principal direction of restricted water proton movement [fractional anisotropy (FA)] and the more general molecular diffusion rate (mean diffusivity; Soares et al., 2013). In voxels with multiple and crossing fiber bundles (i.e., complex fiber populations), DTI cannot readily assign differences in white matter to a specific pathway (Douaud et al., 2011; Jones et al., 2013; Mito et al., 2018). Because up to $90 \%$ of white matter voxels have complex fiber populations (Jones et al., 2013), the calculation of only one averaged direction per voxel renders the diffusion tensor model challenging to interpret biologically (Mito et al., 2018).

To resolve multiple fiber populations within voxels, higherorder diffusion-weighted models have led to a fixel-based analysis (FBA) approach that exploits the fact that DWI images contain information on the presence of multiple fiber bundles (fixels) within a voxel (Raffelt et al., 2017). The approach describes changes in fiber density (FD) and fiber-bundle cross section (FC) and a combination of fiber density and cross section (FDC; Raffelt et al., 2017; Mito et al., 2018). FD reflects microstructural changes in a fiber pathway, with a reduction indicating a decrease in axonal density. FC relates to macrostructural changes of fiber pathways, with a reduction indicating white matter fiber bundle cross-sectional atrophy or preexisting differences in fiber bundle morphology. Last, the FDC measure integrates both the degradation of a fiber tract (FC) and the state of the remaining fibers in a tract (FD; Raffelt et al., 2017; Mito et al., 2018). In summary, the fixel-based analysis approach is tract specific and has a biologically meaningful interpretation.

To date, there have been few diffusion-weighted imaging studies that investigated the effect of tinnitus and acquired hearing loss on the central auditory pathway (Tarabichi et al., 2017). Our study aimed to investigate differences in the largest white matter tract of the central auditory system, the acoustic radiation, connecting the auditory thalamus with the auditory cortex. Two groups with hearing loss, with and without tinnitus, were compared with a normal-hearing control group. Additionally, the relation between fixel-based metrics and demographics, hearing level, and tinnitus-related variables was investigated. Finally, the results from conventional voxel-based diffusion tensor analysis were compared with those of fixel-based analysis.

\section{Materials and Methods}

Ethical approval. This study was performed with the approval of the medical ethical committee of the University Medical Center of Groningen, The Netherlands. Participants signed an informed consent form before participation and received reimbursement for their time and travel costs.

Participants. This study included three groups: participants with hearing loss without tinnitus, participants with hearing loss and tinnitus, and a healthy control group. A total of 93 participants were included: 34 participants with hearing loss and tinnitus, 23 with hearing loss but without tinnitus, and 36 healthy controls. Hearing loss participants had to have bilateral symmetrical hearing loss and normal thresholds up to $1 \mathrm{kHz}$. Pure tone thresholds were obtained in a sound-attenuating booth for frequencies between 0.250 and $8 \mathrm{kHz}$. At the time of inclusion, none of the participants used a hearing aid to improve their hearing or used a tinnitus masker to alleviate their tinnitus. The tinnitus had to be present for at least 6 months. Tinnitus variables collected were laterality, pitch, and the duration of tinnitus. All participants were requested to complete the Hospital Anxiety and Depression Scale (HADS; Zigmond and Snaith, 1983) and the Hyperacusis Questionnaire (HQ; Khalfa et al., 2002). Additionally, the tinnitus participants were requested to fill in the Tinnitus Handicap Inventory (THI; McCombe et al., 2001) and the Tinnitus Reactions Questionnaire (Wilson et al., 1991).

Data acquisition. Diffusion-weighted imaging data were acquired using a Philips Intera 3.0T scanner, with a 32-channel SENSE head coil. All participants were scanned using the following parameters: TR $9000 \mathrm{~ms}$; TE $60.6 \mathrm{~ms}$; voxel size $=2.5 \times 2.5 \times 2.5 \mathrm{~mm}^{3}$; 61 noncollinear gradient directions; 55 slices; $b=1000 \mathrm{~s} / \mathrm{mm}^{2}$; anterior-posterior phase encoding direction.

Preprocessing. First, the data were denoised, motion corrected, and eddy current distortion corrected in FSL (FMRIB Software Library) (Jenkinson et al., 2012). These general preprocessing steps were followed by apparent fiber density-specific preprocessing steps of bias field correction and global intensity normalization across participants. Bias field correction eliminates inhomogeneities in the image that occur because of low spatial frequency intensity areas (Raffelt et al., 2012b). Then, global intensity normalization was performed using group-wise registration. None of the included DWI scans were affected by motion to such an extent that they had to be excluded from the study.

Fixel-based analyses. After preprocessing, fixel-based analyses were performed as outlined in Raffelt et al. (2017); with the aid of MRtrix3 (Tournier et al., 2019). Fixels represent the fiber bundle elements within a voxel and are composed of a set of fibers that are sufficiently similar in orientation. After intensity normalization, single-fiber response functions were estimated for each participant (Tournier et al., 2013). These unique response functions were averaged to obtain a group average response function (Dhollander et al., 2019). The DWI data were upsampled to an isotropic voxel size of $1.3 \mathrm{~mm}$ to increase contrast, and a whole-brain mask was computed from the up-sampled data. Constrained spherical deconvolution was used to estimate the fiber orientation distribution (FOD) for each participant (Dhollander and Connelly, 2016). For the group comparisons, the relevant population templates were created based on a subset of 15 participants per group, in line with the recommendation of the creators of this method (Tournier et al., 2019), ensuring an equal representation of participants of the groups that were investigated. All individual FOD images were then registered to the FOD population template via nonlinear registration (Raffelt et al., 2011, 2012a). The outcome of this registration was used to construct a template mask that contained white matter voxels present in all participants. Subsequently, individual fixels and their orientation in each voxel were identified by segmenting the FODs. All fixel directions were reoriented using the FOD registration warps, and each identified fixel of the template mask was matched to that of the participant image. Finally, FD, FC, and the combination metric FDC were computed.

The interpretations of a reduction in FD, FC, and FDC for the white matter fiber bundle's structural integrity are shown in Figure 1. The FD metric reflects a reduction in the volume of restricted water within a given voxel, which can be because of axonal loss. FD is measured on a 
within-voxel level and reflects the state of the remaining white matter tissue. The FC metric reflects a change in the cross-sectional area occupied by a white matter fiber bundle and can reflect cross-sectional atrophy or acquired axonal loss, such as in Alzheimer's disease (Mito et al., 2018). FDC is a metric that combines the FD and FC metrics. All three metrics can reflect the information-carrying capacity of the white matter tissue (Raffelt et al., 2017). To indicate the magnitude of the significant effects in the groupwise comparisons, the effect size of the FBA outcomes was expressed as a percentage decrease in the FBA metric of one group relative to another group.

Whole-brain probabilistic fiber tractography was used to derive information on local fixel connectivity between neighboring fixels and subsequently apply connectivity-based fixel enhancement to aid statistical analysis (Raffelt et al., 2015). After obtaining the whole-brain tractogram, the initially estimated 20 million tracks were reduced to 2 million to decrease tractogram density biases, using spherical-deconvolutioninformed filtering of tractograms (Smith et al., 2013).

Acoustic radiation fiber tracking. The focus of the current study was on the white matter changes within the acoustic radiation. The population FOD template was used to perform probabilistic tracking of the acoustic radiation to investigate group differences in the white matter between the auditory thalamus [medial geniculate body (MGB)] and the primary auditory cortex. First, masks for the auditory cortex and auditory radiations were derived from the Juelich Histologic atlas in MNI space, followed by the nonlinear registration of the created masks to the population template space using the Non-linear Image Registration Tool of FSL. Then, the bilateral MGB regions of interest were identified by drawing a $4 \mathrm{~mm}$ sphere on the FOD template [MNI coordinates left MGB $(-13.4,-3.1$, 7.3); right MGB $(13.4,-3.6,-7.3)]$. Subsequently, tracking of the acoustic radiations in template space was performed by generating 5000 streamlines, with the MGB as seed ROI and the primary auditory cortex as inclusion ROI. The acoustic radiation masks derived from the Juelich Histologic atlas were used to constrain the fiber tracking anatomically.

Voxel-based metrics. To facilitate the comparison of the FBA metrics with the more conventional DTI-derived metrics, voxel-derived tensorbased analyses were performed on the white matter of the acoustic radiation. The two tensor-based metrics computed for each participant were fractional anisotropy and mean diffusivity (MD), derived with MRtrix3. These tensor metrics were transformed to the population template space with the warps earlier calculated for fixel-based analysis. Voxel-masks were constructed for the tracked acoustic radiations (both left and right) to perform voxel-based diffusion tensor analyses in a manner similar to the fixel-based analyses.

Statistical analysis. Differences among the groups on demographical variables and questionnaire scores were tested for significance using IBM SPSS version 26 software. Group differences for the variable sex were tested with a $\chi^{2}$ test of independence. A three-group ANOVA was used to test for group difference for the variable age, followed by independent pairwise $t$ tests. A Kruskal-Wallis test was used to test for significant differences between questionnaire scores and hearing thresholds, and this test was followed by a pairwise Mann-Whitney $U$ test. Distributions of hearing thresholds and questionnaire scores were assessed by visual inspection. If the dependent variable distributions were similar for both groups, the differences in medians were reported with the Mann-Whitney $U$ test. However, if the distributions were dissimilar, the Mann-Whitney $U$ test was used to investigate the difference in distribution. A Pearson's momentproduct correlation was run to assess the relationship between age and high-frequency hearing loss (quantified as the mean of the thresholds at 4.6 and $8 \mathrm{kHz}$ ).
Group differences in the derived fixel-based metrics (FD, FC, and FDC) were tested for significance with a general linear model. Sex and age were added to the model as covariates. The fixel data were smoothed based on the sparse fixel-fixel connectivity matrix derived from the whole-brain streamline tractogram (Raffelt et al., 2015). For each fixel, a familywise error (FWE) corrected $p$ value was obtained via permutation testing $(n=5000)$. For the visualization of the significant fixels, the mrview tool in MRtrix 3 was used. The significant fixels $(\mathrm{FWE}<0.05)$ were then displayed on the whole-brain tractogram and visualized as streamlines. The effect size of the significant FBA metrics was expressed as a percentage difference. Additionally, individual tract-averaged FD and FC metrics were calculated for the acoustic radiation. This approach allowed us to correlate the fixel-based metrics with age, sex, hearing level (defined as the average of 1,2 , and $4 \mathrm{kHz}$ ), and questionnaire scores.

Threshold free cluster enhancement with permutation testing was used to determine if group differences were significant for the voxelbased tensor derived analysis. Differences in FA and MD metrics were computed on a voxel-by-voxel basis, corrected for sex and age.

\section{Results}

In total, the diffusion-weighted imaging data of 93 participants are presented, along with their demographical variables and questionnaire scores (Table 1). A significantly larger percentage of male participants was present in the hearing-loss group with tinnitus compared with the control group $\left[\chi^{2}(1)=8.6\right.$, $p=0.003]$. There were no significant differences in the percentage of male participants in the hearing-loss group without tinnitus and the control group $\left[\chi^{2}(1)=0.67, p=0.41\right]$, or between both hearing-loss groups $\left[\chi^{2}(1)=3.4, p=0.07\right]$. There were no significant differences in age $(t=1.88, p=0.13)$ between the hearing-loss groups, with and without tinnitus. Both the hearing-loss group with tinnitus $(t=5.32, p<0.001)$ and the hearing-loss group without tinnitus $(t=5.97, p<0.001)$ were significantly older than those in the control group. The pure tone audiograms, averaged over both ears, are reported for the three groups in Figure 2. On average, the hearing-loss group with tinnitus had higher thresholds than the hearing group without tinnitus. These differences did not reach significance after correction for multiple comparisons (Fig. 2). Both hearing-loss groups had significantly higher thresholds than the control group for all frequencies tested. 
Table 1. Demographics and questionnaire scores of the three participant groups

\begin{tabular}{llll}
\hline $\begin{array}{l}\text { Groups } \\
\text { Questionnaires }\end{array}$ & Controls & Hearing loss & $\begin{array}{l}\text { Hearing } \\
\text { loss }+ \text { tinnitus }\end{array}$ \\
\hline $\begin{array}{l}\text { Demographics } \\
\text { Sex }\end{array}$ & $N=36$ & $N=23$ & $N=34$ \\
$\begin{array}{l}\text { Mean age years } \\
\quad \text { (age range) }\end{array}$ & 18 male, 18 female & 14 male, 9 female & 27 male, 7 female*** \\
HADS Anxiety & $55 \pm 14(18-67)$ & $62 \pm 8(33-75)^{*}$ & $60 \pm 8(41-72)^{*}$ \\
HADS Depression & $3 \pm 3(0-9)$ & $2 \pm 3(0-10)$ & $4 \pm 4(0-16)^{* *}$ \\
HQ & $11 \pm 7(0-27)$ & $9 \pm 5(1-18)$ & $16 \pm 8(0-30)^{* *}$ \\
THI & & & $39 \pm 19(16-82)$ \\
Tinnitus & & & \\
Mean duration (years) & & & $14 \pm 9(1-33)$ \\
Tinnitus pitch & & & $1-<4 \mathrm{kHz}(n=9)$ \\
& & & $4-7 \mathrm{kHz}(n=12)$ \\
& & & $\geq 8 \mathrm{kHz}(n=11)$ \\
& & & Broadband $(n=2)$
\end{tabular}

*Indicates that groups differed significantly from the control group; ${ }^{*}$ indicates that hearing-loss groups differed significantly from one another at $p<0.001, \chi^{2}$, ANOVA, Kruskal-Wallis, and Mann-Whitney, respectively.

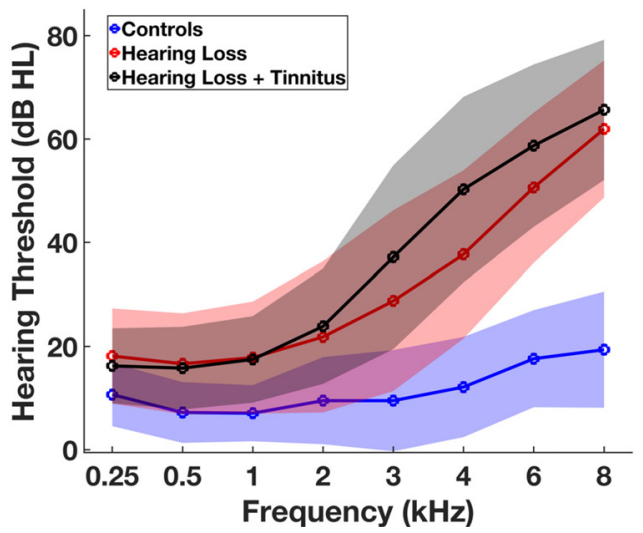

Figure 2. Hearing thresholds for the three groups with their respective SDs. After correction for multiple comparisons, the median thresholds of the hearing-loss groups were not significantly different $(250 \mathrm{~Hz}, p=0.60 ; 500 \mathrm{~Hz}, p=0.95 ; 1 \mathrm{kHz}, p=0.83 ; 2 \mathrm{kHz}, p=0.90$; $3 \mathrm{kHz}, p=0.09 ; 4 \mathrm{kHz}, p=0.04 ; 6 \mathrm{kHz}, p=0.17 ; 8 \mathrm{kHz}, p=0.42$ ). However, at $4 \mathrm{kHz}$, the difference in hearing thresholds was significant at an uncorrected level of $p<0.05$ $(p=0.04)$. Both hearing-loss groups, with and without tinnitus, differed significantly on all frequencies from the control group. Higher median thresholds were present in the hearingloss group without tinnitus $(250 \mathrm{~Hz}, p=0.001 ; 500 \mathrm{~Hz}-8 \mathrm{kHz},=p<0.0005)$ and the hearing-loss group with tinnitus $(250 \mathrm{~Hz}, p=0.002 ; 500 \mathrm{~Hz}-8 \mathrm{kHz}=p<0.0005)$, compared with controls.

There was a significant positive correlation between age and high-frequency hearing loss $\left(r_{(91)}=0.629, p<0.001\right)$. Furthermore, the hearing-loss group with tinnitus had significantly higher scores on the Hyperacusis Questionnaire $(U=96.5, z=-2.89 p=0.004)$ and the depression scale of the HADS $(U=126.5, \mathrm{z}=-2.41$, $p=0.016)$ compared with the hearing-loss group without tinnitus, at a level corrected for multiple comparisons.

\section{Tract of interest fixel-based analysis}

The auditory radiation was tracked from the medial geniculate body to the primary auditory cortex. The outcomes presented in this study are based on the metrics of these tracts, which are constructed based on 5000 streamlines. These tracts are shown in Figure 3; the left acoustic radiation is presented in blue and the right in red. The FD, FC, and the FDC of the three groups were

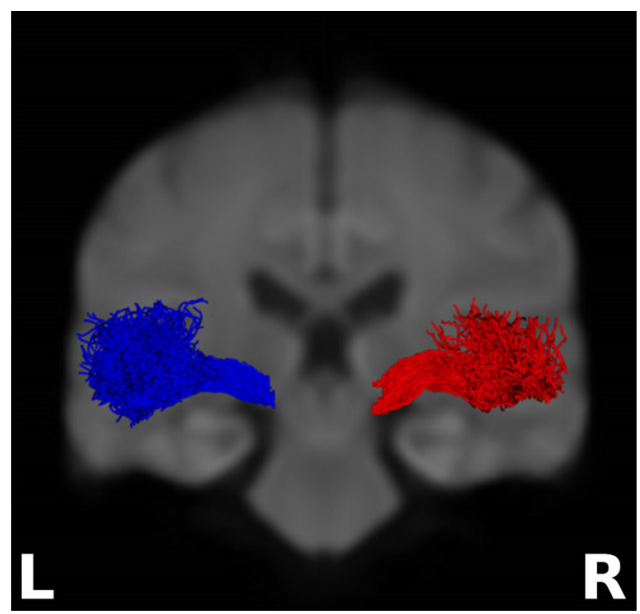

Figure 3. Tracked acoustic radiations for left (blue) and right (red) hemispheres. The tracts are overlaid on a representative coronal slice. Tracking was performed per hemisphere from the MGB to the primary auditory cortex (TE 1.0).

compared, with the variables of age and sex controlled for within the model. This analysis identified a significantly smaller FC of the auditory radiation in hearing loss with tinnitus compared with the control group. This effect was significant for the left auditory radiation $(p=0.024)$, and approached significance for the right auditory radiation $(p=0.056)$. Expressed as a percentage change of the hearing-loss group with tinnitus compared with the group mean of the control group, this corresponds to a decrease in FC of $5.4 \%$. There were no other significant group differences for the FC, FD, and FDC metrics.

The significant difference in FC occurs in the area of the auditory radiation closest to the medial geniculate area of the thalamus, shown in Figure 4. The black lines represent all the fixels identified within the auditory radiation mask at this brain slice (Fig. 4A). The colored lines represent the fixels where a significant difference was detected between the hearing-loss group with tinnitus and the control group. The enlarged inset illustrates that even in voxels with crossing fibers (multiple fixels in a single voxel), the FBA method identifies significant differences among the groups only in the fixels belonging to the acoustic radiation, which runs from medial to lateral, that is, from the thalamus in the direction of the auditory cortex (Fig. $4 B-D$ ). The streamline segments that correspond to the significant fixels (FWE-corrected $p$ value $<0.05$ ) of the FC are displayed in Figure 5.

\section{Association tinnitus-related variables, questionnaire scores, hearing level, sex, and age with fiber density and cross- section metrics}

As summarized in Table 2, there was a significant association between age and fiber density for both the left and the right acoustic radiation. There was no significant association between age and fiber cross section. Furthermore, there was no significant association between either the fiber density or fiber cross-section metrics and the THI score, sex, HADS score, or any of the tinnitus characteristics such as tinnitus pitch, duration, lateralization, or hearing level, at a level corrected for multiple comparisons $(\mathrm{FWE}<0.05)$. Because the FDC metric is a combination of the FD and FC metrics, we did not include it in this analysis.

To test whether the higher HQ scores in the group with tinnitus affected the results, we split the tinnitus group at an HQ score of 22 (Aazh and Moore, 2017; Koops and van Dijk, 2021) and compared the FC and FD metrics of the two resulting groups (high 


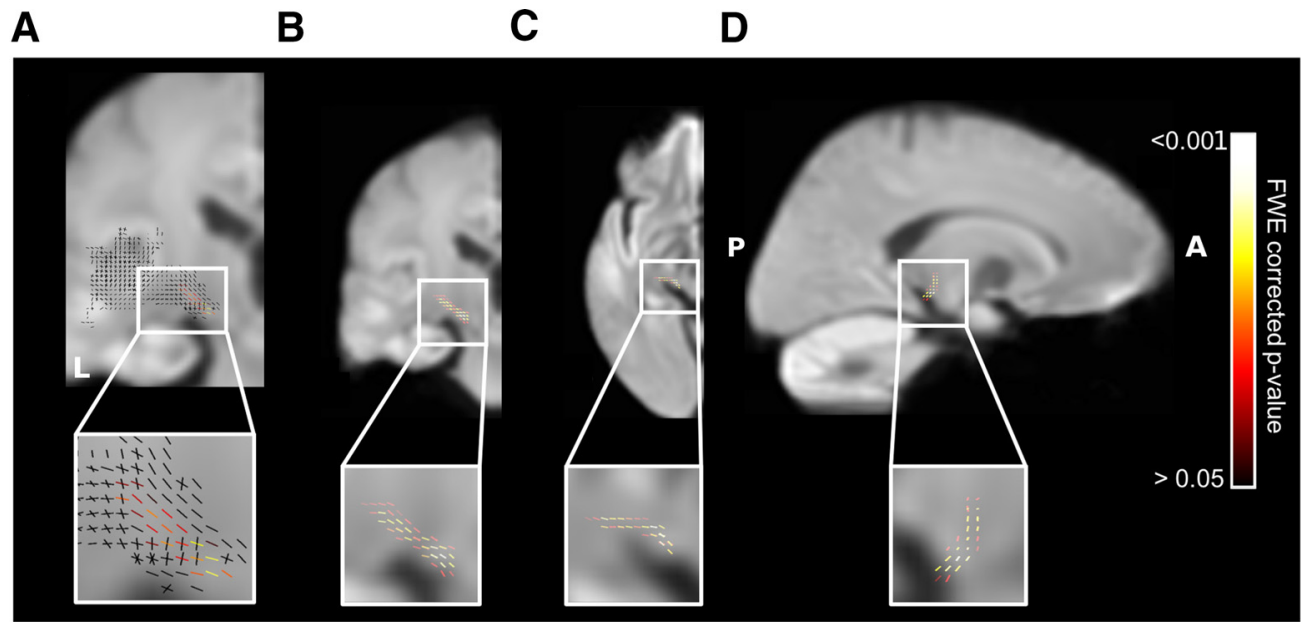

Figure 4. Region of the left acoustic radiation with an altered cross-sectional metric in participants with hearing loss and tinnitus compared with controls. $\boldsymbol{A}$, The line segments indicate fixels; each fixel corresponds to a population of fibers that pass through the corresponding voxel. The line segments represent all the fixels that were identified within the mask of left the acoustic radiation. The upper portion is an overview to indicate the anatomic location. The zoomed-in inset shows the fixels that belong to a tract with a significantly smaller cross section in the hearing-loss group with tinnitus than in the control group. These significantly different fixels are color-coded by their respective FWE-corrected $p$ values. For black fixels, there was no significant difference between the groups. $\boldsymbol{B}$, The nonsignificant fixels are omitted here. Only the fixels with a significantly decreased cross section are projected on a coronal slice; $\boldsymbol{C}$, An axial slice; and $\boldsymbol{D}$, A sagittal slice. Significant differences can be observed in the area of the auditory radiation closest to the medial geniculate area of the thalamus. Note that the FBA identified significant differences in fixels that are part of the acoustic radiation only. Crossing fixels were not significantly different between the groups and are therefore shown in black. All panels show one representative brain slice with the projected fixels. $\boldsymbol{D}$, The small line segments indicate that fixels, and thus the corresponding nerve fibers, are oriented out of the depicted sagittal plane.

and low HQ scores) with two-sample $t$ tests. There were no significant or near significant differences in average FC (left, $p=0.187$; right, $p=0.150$ ) or FD (left, $p=0.678$; right, $p=0.904$ ) of the acoustic radiation between participants with hearing loss and tinnitus with high and low HQ scores. It thus appears that although the presence of tinnitus is related to an altered FC of the acoustic radiation, additional hyperacusis does not alter this relation.

\section{Voxel-based metrics of tract of interest}

A significant increase in MD in the left acoustic radiation was observed for the hearing-loss group with tinnitus, compared with the control group $(p=0.03$, FWE corrected). No significant or near significant differences in fractional anisotropy (FA) or mean diffusivity (MD) were observed for any other group comparisons. An overlay of the voxels with significantly different mean diffusivity on the fixel mask with the FC metric shows an anatomic overlap between the voxels with increased MD and the fixels with decreased FC. The voxels with a significant increase in MD are located close to the medial geniculate body and slightly more anterior to the fixels with a significant decrease in FC (Fig. 6).

\section{Discussion}

Fixel-based analysis was used to investigate differences in the acoustic radiation in hearing-impaired participants, with and without tinnitus. In the presence of tinnitus, a significant reduction in the acoustic radiation's cross section was observed for the left acoustic radiation. Conventional DTI showed a significant increase in mean diffusivity at a similar location in the left acoustic radiation of hearing-loss participants with tinnitus compared with controls.

\section{Comparison with previous DWI studies in acquired hearing loss}

Previous reports on voxel-based tensor metrics in acquired hearing loss or presbycusis are sparse. Only two studies included

regions of interest in the auditory pathway or reported on its white matter tracts. Two whole-brain voxel-based studies were identified. One reported increased MD in the transverse temporal plane and decreased FA for the middle temporal gyrus (Ma et al., 2016). Because no segmentation or masking of white matter was reported, gray matter may have affected this report's voxelbased measures. The second study reported decreased FA for the anterior thalamic radiation, specified as acoustic radiation (Husain et al., 2011). In contrast, a diffusion tensor tractography study reported no significant changes in FA or MD in the auditory pathway of hearing-loss participants (Profant et al., 2014). In line with the latter study, our tractography results do not indicate significant differences in FA or MD related to hearing loss. Similarly, no differences related to hearing loss in the FD or FC of the acoustic radiation were identified. Whereas earlier studies used voxel-based tensor metrics to infer differences, FBA allows us to firmly place the significant fixels in the fiber tract of interest. Thus, neither conventional DTI nor the improved fiber determination of 
Table 2. Relation between fixel-based metrics and age, hearing loss, and tinnitus-related variables

\begin{tabular}{|c|c|c|c|c|c|}
\hline & & \multicolumn{2}{|c|}{$\begin{array}{l}\text { Left acoustic } \\
\text { radiation }\end{array}$} & \multicolumn{2}{|c|}{$\begin{array}{l}\text { Right acoustic } \\
\text { radiation }\end{array}$} \\
\hline & & $R$ & $p$ & $R$ & $p$ \\
\hline \multicolumn{6}{|l|}{ General variables } \\
\hline \multirow[t]{2}{*}{ Age } & $F D$ & -0.38 & $<0.001^{*}$ & -0.40 & $<0.001^{*}$ \\
\hline & $\log F C$ & -0.07 & 0.53 & -0.06 & 0.60 \\
\hline \multirow[t]{2}{*}{ Hearing } & $F D$ & -0.20 & 0.06 & -0.06 & 0.59 \\
\hline & $\log F C$ & -0.17 & 0.11 & -0.04 & 0.71 \\
\hline \multicolumn{6}{|c|}{ Tinnitus-related variables } \\
\hline \multirow[t]{2}{*}{ Sex } & $F D$ & 0.24 & 0.047 & 0.12 & 0.34 \\
\hline & $\log F C$ & -0.09 & 0.43 & -0.04 & 0.73 \\
\hline \multirow[t]{2}{*}{ THI } & FD & 0.29 & 0.11 & 0.18 & 0.33 \\
\hline & $\log F C$ & -0.003 & 0.99 & -0.15 & 0.41 \\
\hline \multirow[t]{2}{*}{ Duration } & $F D$ & 0.17 & 0.45 & -0.23 & 0.30 \\
\hline & $\log F C$ & 0.21 & 0.34 & -0.27 & 0.23 \\
\hline \multirow[t]{2}{*}{ Pitch } & $F D$ & 0.23 & 0.20 & -0.20 & 0.26 \\
\hline & $\log F C$ & 0.12 & 0.51 & -0.42 & 0.02 \\
\hline \multicolumn{6}{|l|}{ Questionnaires } \\
\hline \multirow[t]{2}{*}{ Laterality } & $F D$ & 0.07 & 0.68 & 0.27 & 0.12 \\
\hline & $\log F C$ & 0.23 & 0.20 & 0.24 & 0.17 \\
\hline \multirow[t]{2}{*}{ HADS Anxiety } & $F D$ & 0.28 & 0.02 & 0.29 & 0.01 \\
\hline & $\log F C$ & 0.12 & 0.32 & 0.06 & 0.59 \\
\hline \multirow[t]{2}{*}{ HADS Depression } & $F D$ & 0.18 & 0.13 & 0.13 & 0.26 \\
\hline & $\log F C$ & 0.14 & 0.26 & 0.08 & 0.53 \\
\hline
\end{tabular}

There was a significant negative correlation between age and fiber density for both the left and the right acoustic radiation (FWE-corrected $p$ value $<0.05$ ), indicated in bold. Hearing level, indicated by the mean of 1,2 , and $4 \mathrm{kHz}$ did not significantly correlate with either FD or FC. The type of fiber-based metric is indicated in italic. Tinnitus-related variables and questionnaire scores were not significantly associated with FD or FC fixel-based metrics. The asterisks denote a significant relation at a $p$ level corrected for multiple comparisons. The association for sex and laterality with FBA-metrics was tested with a point-biserial correlation. All other associations were tested with a Pearson's correlation test. *Bonferroni's.

2014), and one of them reported tinnitus-related significant voxels in the white matter of the auditory pathway (SeydellGreenwald et al., 2014). The data provided in these articles cannot ascertain if the results are specifically located in the acoustic radiation or reflect differences in fiber paths that run in close proximity, such as the longitudinal fasciculus or the anterior thalamic radiation. The application of fiber tractography can give a better indication of the specific tracts. A fiber tractography study reported no significant differences in the voxel-based tensorderived metrics in a group with tinnitus and hearing loss compared with a control group (Crippa et al., 2010). Although the former study did not include age and sex in its statistical model, in the current study we did correct for differences in age and sex. In the present study, we identified a difference in MD between the hearing-loss group with tinnitus and controls, but there were no significant differences between the hearing-loss groups with and without tinnitus. Overall, our findings suggest that in the presence of tinnitus, there is a more pronounced increase in MD in the acoustic radiation, near the medial geniculate body, than in hearing loss without tinnitus.

\section{Reduction of fiber-bundle cross section and increase of mean diffusivity in tinnitus}

A significant reduction in FC was observed in the left acoustic radiation of participants with hearing loss and tinnitus. A similar effect, although nonsignificant, was observed in the right acoustic radiation. The increase in mean diffusivity (MD) partially overlapped in location with the reduction in FC (Fig. 6). Although we observed a loss of restriction of free diffusion, there was no loss of directionality of this diffusion. The decrease in FC may reflect fiber bundle atrophy or poor myelination of the axons within this area of the acoustic radiation. The degradation of myelination is not expected to yield a drastic decrease in FA as even the complete absence of myelin decreases FA only by $\sim 20 \%$. However, the absence of myelination causes an increase in MD of $\sim 50 \%$ (Gulani et al., 2001). Thus, the FC reduction and $\mathrm{MD}$ increase in tinnitus both suggest disruption or thinning of the myelin sheet. The confinement of the observed effect to the thalamic end of the acoustic radiation can reflect that at more distal points along this tract; the axons are less densely packed as the fibers fan out to project to the larger area of the auditory cortex.

\section{Myelination, white matter} reductions, and evoked potentials in tinnitus

Animal research suggested that tinnitus is related to a loss of inner hair cell ribbons (Rüttiger et al., 2013). These ribbons are the electron-dense struc-

FBA identified significant differences related to acquired hearing loss in the acoustic radiation tracts.

\section{Comparison with previous DWI studies on tinnitus}

Diffusion imaging studies on tinnitus that included a control group and reported on or included white matter tracts of the auditory pathway are sparse. Two studies implemented wholebrain analyses (Husain et al., 2011; Seydell-Greenwald et al., tures associated with presynaptic active zones at the inner hair cell base. A loss of these ribbons would disrupt the sustained release of neurotransmitters at the first auditory synaptic junction (Matthews and Fuchs, 2010) and thereby have an impact on auditory processing. Such damage may have caused the small but not significantly higher average thresholds of the hearing-loss group with tinnitus (Fig. 2). Furthermore, it has been suggested 
that tinnitus may relate to a loss of specific auditory nerve fibers. On the one hand, the loss of low-spontaneous rate fibers (lowSRFs) has been indicated in tinnitus (Schaette and McAlpine, 2011). However, recent evidence suggests that tinnitus in normal hearing doesn't correspond to diminished speech in noise perception (Zeng et al., 2020), which would be expected with the loss of low-SRFs. On the other hand, the specific loss of fast-coding high-spontaneous rate fibers (high-SRFs) has also been implicated in tinnitus (Bauer et al., 2007; Knipper et al., 2020), as are a combination of both fiber types (Paul et al., 2017). The high-SRFs have a thicker myelin sheet than low- and mediumSRFs (Liberman and Oliver, 1984; Gleich and Wilson, 1993), facilitating fast information transfer. This is vital to the functionality of the auditory system as it relies on precise timing. Future work could assess whether damage to specific auditory nerve fibers instigates a Wallerian-like degeneration process that leads to cross-sectional atrophy and increased medial diffusivity in the central auditory tracts.

Overall, myelination in the CNS is enhanced by neuronal activity (Demerens et al., 1996), and a reduction in sound activity has been related to a decrease in the myelin thickness and the number of large-diameter axons in the central auditory system (Sinclair et al., 2017). Previously, our group and others have shown that the auditory cortex is less responsive to high-frequency sounds in tinnitus participants with mild or moderate hearing loss than in those without tinnitus (Hofmeier et al., 2018; Koops et al., 2020). The reduction in the acoustic radiation's cross section in the presence of tinnitus can relate to the previously reported reduction in sound-evoked activity in tinnitus. Critical damage at the peripheral level could result in a thinning of the myelin sheet of the corresponding central auditory fibers that are no longer stimulated as a consequence. The additional presence of tinnitus in hearing loss may enlarge this effect.

Auditory brainstem responses (ABR) can detect changes in the amplitude and time course of an auditory signal traveling from peripheral to more central auditory areas via the white matter tracts. In tinnitus, the most consistently reported findings are reduced (early) ABR amplitudes (Milloy et al., 2017; Hofmeier et al., 2018) and prolonged wave I and V latencies (Ikner and Hassen, 1990; Ravikumar and Ashok Murthy, 2016; Hofmeier et al., 2018), similar to the changes observed in high-frequency hearing loss (Sand and Saunte, 1994; Watson, 1996; Lewis et al., 2015). A loss of high-SRFs at the peripheral level, a suggested hallmark of tinnitus, could explain the reported ABR wave latency prolongation (Knipper et al., 2020), which may relate to a reduction in myelination (Kovach et al., 1999). Together, the previously reported tinnitus-related $A B R$ features and the current findings of cross-sectional atrophy in the acoustic radiation point toward reduced myelination of the peripheral and central auditory pathway in tinnitus, which warrants further investigation.

\section{Age-related decline of fiber density}

FD of the acoustic radiation and age were negatively correlated, indicating a progressive loss of thalamocortical axons with increasing age. Consequently, signal transfer from the thalamic medial geniculate body to the primary auditory cortex may be disturbed. Older adults with hearing loss perform worse than younger people with similar hearing loss (Dubno et al., 1984; Fitzgibbons and Gordon-Salant, 1995; Pichora-Fuller et al., 1995; Wingfield et al., 2006; Cardin, 2016), and the loss of axonal density with age may contribute to the poorer speech understanding observed in older adults. A minor decrease in peripheral myelination has previously been related to dramatic degradation of fine temporal structure coding, whereas it hardly affects hearing thresholds (Resnick et al., 2018). Presumably, the reduction of fiber density reported here further contributes to poorer information transfer of auditory information. These findings have implications for the rehabilitation of hearing in older individuals. Peripheral stimulation (i.e., hearing aids) may be supplemented with treatments aimed at stimulating cortical plasticity (e.g., inhibition of neural growth inhibitors in combination with tailored auditory training).

\section{Conclusion}

Our study did not identify significant changes in the acoustic radiation specifically related to acquired hearing loss. On the other hand, the additional presence of tinnitus was related to macrostructural degeneration of the left acoustic radiation near the medial geniculate nucleus. In addition, a significant increase in MD was identified in participants with hearing loss and tinnitus, compared with controls. Both the diffusion tensor model and the fixel-based analysis results point toward a reduction in the axonal myelination of the acoustic radiation in the presence of tinnitus. Together with the peripheral deafferentation reported in animal studies on tinnitus, the current findings suggest impaired integrity of nerve fibers at various levels of the auditory system. Furthermore, age was related to a decrease in fiber density of the acoustic radiation, which relates to axonal loss. This finding suggests a possible relation between acoustic radiation axonal loss and a decline in sound processing in older adults. This latter finding may have implications for the rehabilitation approaches in older individuals.

\section{References}

Aazh H, Moore BCJ (2017) Factors related to uncomfortable loudness levels for patients seen in a tinnitus and hyperacusis clinic. Int J Audiol 56:793800 .

Bauer CA, Brozoski TJ, Myers K (2007) Primary afferent dendrite degeneration as a cause of tinnitus. J Neurosci Res 85:1489-1498.

Cardin V (2016) Effects of aging and adult-onset hearing loss on cortical auditory regions. Front Neurosci 10:199.

Crippa A, Lanting CP, Dijk P. v, Roerdink JBT (2010) A diffusion tensor imaging study on the auditory system and tinnitus. Open Neuroimag J 4:16-25.

Demerens C, Stankoff B, Logak M, Anglade P, Allinquant B, Couraud F, Zalc B, Lubetzki C (1996) Induction of myelination in the central nervous system by electrical activity. Proc Natl Acad Sci U S A 93:9887-9892.

Dhollander T, Connelly A (2016) A novel iterative approach to reap the benefits of multi-tissue CSD from just single-shell $(+b=0)$ diffusion MRI data. Proceedings of the 24th annual meeting of the International Society of Magnetic Resonance in Medicine, pp 3010.

Dhollander T, Mito R, Raffelt D, Connelly A (2019) Improved white matter response function estimation for 3-tissue constrained spherical deconvolution. Paper presented at the 27th annual meeting of the International Society of Magnetic Resonance in Medicine, Montréal, Québec, Canada, May.

Douaud G, Jbabdi S, Behrens TEJ, Menke RA, Gass A, Monsch AU, Rao A, Whitcher B, Kindlmann G, Matthews PM, Smith S (2011) DTI measures in crossing-fibre areas: increased diffusion anisotropy reveals early white matter alteration in MCI and mild Alzheimer's disease. Neuroimage 55:880-890.

Dubno JR, Dirks DD, Morgan DE (1984) Effects of age and mild hearing loss on speech recognition in noise. J Acoust Soc Am 76:87-96.

Fitzgibbons PJ, Gordon-Salant S (1995) Age effects on duration discrimination with simple and complex stimuli. J Acoust Soc Am 98:3140-3145.

Gleich O, Wilson S (1993) The diameters of guinea pig auditory nerve fibres: distribution and correlation with spontaneous rate. Hear Res 71:69-79.

Gulani V, Webb AG, Duncan ID, Lauterbur PC (2001) Apparent diffusion tensor measurements in myelin-deficient rat spinal cords. Magn Reson Med 45:191-195.

Hofmeier B, Wolpert S, Aldamer ES, Walter M, Thiericke J, Braun C, Zelle D, Rüttiger L, Klose U, Knipper M (2018) Reduced sound-evoked and 
resting-state BOLD fMRI connectivity in tinnitus. NeuroImage Clin 20:637-649.

Husain FT, Medina RE, Davis CW, Szymko-Bennett Y, Simonyan K, Pajor NM, Horwitz B (2011) Neuroanatomical changes due to hearing loss and chronic tinnitus: a combined VBM and DTI study. Brain Res 1369:74-88.

Ikner CL, Hassen AH (1990) The effect of tinnitus on ABR latencies. Ear Hear 11:16-20.

Jenkinson M, Beckmann CF, Behrens TEJ, Woolrich MW, Smith SM (2012) FSL. Neuroimage 62:782-790.

Jones D, Knösche T, Turner R (2013) White matter integrity, fiber count, and other fallacies: the do's and don'ts of diffusion MRI. Neuroimage 73:239-254.

Khalfa S, Dubal S, Veuillet E, Perez-Diaz F, Jouvent R, Collet L (2002) Psychometric normalization of a hyperacusis questionnaire. ORL J Otorhinolaryngol Relat Spec 64:436-442.

Knipper M, van Dijk P, Schulze H, Mazurek B, Krauss P, Scheper V, Warnecke A, Schlee W, Schwabe K, Singer W, Braun C, Delano PH, Fallgatter AJ, Ehlis AC, Searchfield GD, Munk MHJ, Baguley DM, Rüttiger L (2020) The neural bases of tinnitus: lessons from deafness and cochlear implants. J Neurosci 40:7190-7202.

Koops EA, van Dijk P (2021) Hyperacusis in tinnitus patients relates to enlarged subcortical and cortical responses to sound except at the tinnitus frequency. Hear Res 401:108158.

Koops EA, Renken RJ, Lanting CP, Dijk P. v (2020) Cortical tonotopic map changes in humans are larger in hearing loss than in additional tinnitus. J Neurosci 40:3178-3185.

Kovach MJ, Lin JP, Boyadjiev S, Campbell K, Mazzeo L, Herman K, Rimer LA, Frank W, Llewellyn B, Jabs EW, Gelber D, Kimonis VE (1999) A unique point mutation in the PMP22 gene is associated with CharcotMarie-Tooth disease and deafness. Am J Hum Genet 64:1580-1593.

Lewis JD, Kopun J, Neely ST, Schmid KK, Gorga MP (2015) Tone-burst auditory brainstem response wave $\mathrm{V}$ latencies in normal-hearing and hearing-impaired ears. J Acoust Soc Am 138:3210-3219.

Liberman MC, Oliver ME (1984) Morphometry of intracellularly labeled neurons of the auditory nerve: correlations with functional properties. J Comp Neurol 223:163-176.

Ma W, Li M, Gao F, Zhang X, Shi L, Yu L, Zhao B, Chen W, Wang G, Wang X (2016) DTI analysis of presbycusis using voxel-based analysis. AJNR Am J Neuroradiol 37:2110-2114.

Matthews G, Fuchs P (2010) The diverse roles of ribbon synapses in sensory neurotransmission. Nat Rev Neurosci 11:812-822.

McCombe A, Baguley D, Coles R, McKenna L, McKinney C, Windle-Taylor P. (2001) Guidelines for the grading of tinnitus severity: the results of a working group commissioned by the British Association of Otolaryngologists, Head and Neck Surgeons, 1999. Clin Otolaryngol Allied Sci 26:388-393.

McCormack A, Edmondson-Jones M, Somerset S, Hall D (2016) A systematic review of the reporting of tinnitus prevalence and severity. Hear Res 337:70-79.

Milloy V, Fournier P, Benoit D, Noreña A, Koravand A (2017) Auditory brainstem responses in tinnitus: a review of who, how, and what? Front Aging Neurosci 9:237.

Mito R, Raffelt D, Dhollander T, Vaughan DN, Tournier J-D, Salvado O, Brodtmann A, Rowe CC, Villemagne VL, Connelly A (2018) Fibre-specific white matter reductions in Alzheimer's disease and mild cognitive impairment. Brain 141:888-902.

Paul BT, Bruce IC, Roberts LE (2017) Evidence that hidden hearing loss underlies amplitude modulation encoding deficits in individuals with and without tinnitus. Hear Res 344:170-182.

Pichora-Fuller MK, Schneider BA, Daneman M (1995) How young and old adults listen to and remember speech in noise. J Acoust Soc Am 97:593-608.

Profant O, Škoch A, Balogová Z, Tintěra J, Hlinka J, Syka J (2014) Diffusion tensor imaging and MR morphometry of the central auditory pathway and auditory cortex in aging. Neuroscience 260:87-97.

Raffelt D, Tournier JD, Fripp J, Crozier S, Connelly A, Salvado O (2011) Symmetric diffeomorphic registration of fibre orientation distributions. Neuroimage 56:1171-1180.

Raffelt D, Tournier J-D, Crozier S, Connelly A, Salvado O (2012a) Reorientation of fiber orientation distributions using apodized point spread functions. Magn Reson Med 67:844-855.
Raffelt D, Tournier JD, Rose S, Ridgway GR, Henderson R, Crozier S, Salvado O, Connelly A (2012b) Apparent fibre density: a novel measure for the analysis of diffusion-weighted magnetic resonance images. Neuroimage 59:3976-3994.

Raffelt DA, Smith RE, Ridgway GR, Tournier JD, Vaughan DN, Rose S, Henderson R, Connelly A (2015) Connectivity-based fixel enhancement: whole-brain statistical analysis of diffusion MRI measures in the presence of crossing fibres. Neuroimage 117:40-55.

Raffelt DA, Tournier J-D, Smith RE, Vaughan DN, Jackson G, Ridgway GR, Connelly A (2017) Investigating white matter fibre density and morphology using fixel-based analysis. Neuroimage 144:58-73.

Ravikumar G, Ashok Murthy V (2016) A study of brainstem auditory evoked responses in normal hearing patients with tinnitus. Indian J Otolaryngol Head Neck Surg 68:429-433.

Resnick JM, O’Brien GE, Rubinstein JT (2018) Simulated auditory nerve axon demyelination alters sensitivity and response timing to extracellular stimulation. Hear Res 361:121-137. ]

Rüttiger L, Singer W, Panford-Walsh R, Matsumoto M, Lee SC, Zuccotti A, Zimmermann U, Jaumann M, Rohbock K, Xiong H, Knipper M (2013) The reduced cochlear output and the failure to adapt the central auditory response causes tinnitus in noise exposed rats. PLoS One 8:e57247.

Sand T, Saunte C (1994) ABR amplitude and dispersion variables: relation to audiogram shape and click polarity. Scand Audiol 23:7-12.

Schaette R, McAlpine D (2011) Tinnitus with a normal audiogram: physiological evidence for hidden hearing loss and computational model. J Neurosci 31:13452-13457.

Seydell-Greenwald A, Raven EP, Leaver AM, Turesky TK, Rauschecker JP (2014) Diffusion imaging of auditory and auditory-limbic connectivity in tinnitus: preliminary evidence and methodological challenges. Neural Plast 2014:145943.

Sinclair JL, Fischl MJ, Alexandrova O, $\mathrm{He} \beta$ M, Grothe B, Leibold C, KoppScheinpflug C (2017) Sound-evoked activity influences myelination of brainstem axons in the trapezoid body. J Neurosci 37:8239-8255.

Singer W, Zuccotti A, Jaumann M, Lee SC, Panford-Walsh R, Xiong H, Zimmermann U, Franz C, Geisler H-S, Köpschall I, Rohbock K, Varakina K, Verpoorten S, Reinbothe T, Schimmang T, Rüttiger L, Knipper M (2013) Noise-induced inner hair cell ribbon loss disturbs central arc mobilization: a novel molecular paradigm for understanding tinnitus. Mol Neurobiol 47:261-279.

Smith RE, Tournier J-D, Calamante F, Connelly A (2013) SIFT: spherical-deconvolution informed filtering of tractograms. Neuroimage 67:298-312.

Soares JM, Marques P, Alves V, Sousa N (2013) A hitchhiker's guide to diffusion tensor imaging. Front Neurosci 7:31.

Tarabichi O, Kozin ED, Kanumuri VV, Barber S, Ghosh S, Sitek KR, Reinshagen K, Herrmann B, Remenschneider AK, Lee DJ (2017) Diffusion tensor imaging of central auditory pathways in patients with sensorineural hearing loss: a systematic review. Otolaryngol Head Neck Surg 158:432-442.

Tournier JD, Calamante F, Connelly A (2013) Determination of the appropriate $\mathrm{b}$ value and number of gradient directions for high-angular-resolution diffusion-weighted imaging. NMR Biomed 26:1775-1786.

Tournier J-D, Smith R, Raffelt D, Tabbara R, Dhollander T, Pietsch M, Christiaens D, Jeurissen B, Yeh C-H, Connelly A (2019) MRtrix3 : A fast, flexible and open software framework for medical image processing and visualisation. Neuroimage 202:116137.

Watson DR (1996) The effects of cochlear hearing loss, age and sex on the auditory brainstem response. Audiology 35:246-258. ]

Wilson PH, Henry J, Bowen M, Haralambous G (1991) Tinnitus reaction questionnaire. J Speech Lang Hear Res 34:197-201.

Wingfield A, McCoy SL, Peelle JE, Tun PA, Cox LC (2006) Effects and adult aging and hearing loss on comprehension of rapid speech varying in syntactic complexity. J Am Acad Audiol 17:487-497.

Zeng F-G, Richardson M, Turner K (2020) Tinnitus does not interfere with auditory and speech perception. J Neurosci 40:6007-6017.

Zigmond AS, Snaith RP (1983) The hospital anxiety and depression scale. Acta Psychiatr Scand 67:361-370. 